Check for updates

Cite this: RSC Adv., 2017, 7, 43047

Received 26th July 2017

Accepted 16th August 2017

DOI: $10.1039 / \mathrm{c} 7 \mathrm{ra0} 8260 f$

rsc.li/rsc-advances

\section{Uniform e-beam irradiation-induced athermal straightening of axially curved amorphous $\mathrm{SiO}_{x}$ nanowires}

\author{
Jiangbin Su (D) ab and Xianfang Zhu (iD *a
}

\begin{abstract}
The reshaping of amorphous $\mathrm{SiO}_{x}$ nanowires $\left(\mathrm{a}-\mathrm{SiO}_{x} \mathrm{NWs}\right)$ as solely induced by uniform electron beam (ebeam) irradiation was in situ studied at room temperature using transmission electron microscopy. It was observed that the axially straight NW kept its perfect straight cylinder-like wire shape and demonstrated a uniform radial shrinkage with the increase of irradiation time. In contrast, the axially curved NW quickly became straight accompanied by uniform axial shrinkage and uniform radial expansion. It is expected that such a study in particular on the athermal straightening of axially curved NW has important implications for nanoinstability and nanoprocessing of future NW-based structures or devices. More importantly, the findings demonstrate that the traditional knock-on mechanism and e-beam heating effect are inadequate to explain these processes while our proposed nanocurvature effect and energetic beam-induced athermal activation effect obviously dominate the processes.
\end{abstract}

\section{Introduction}

As one of the important quasi-one dimensional photoluminescence nanomaterials in the blue and near-infrared regions, amorphous $\mathrm{SiO}_{x}$ nanowires $\left(\mathrm{a}-\mathrm{SiO}_{x} \mathrm{NWs}\right)$ have attracted much attention in fields such as scanning near-field optical microscopes, integrated optical devices and photon detectors. ${ }^{1,2}$ However, due to the difficulties in controllable synthesis or assembly, as-prepared a-SiO ${ }_{x}$ NWs may not have ideal wire shapes such as straightness, diameter and length. Furthermore, such glassy silicon oxides are characterized by covalent and ionic bonding and hence brittle materials. They do not exhibit ductility at room temperature to allow plastic deformation after their fabrication into structures or devices. It may thus limit the application of $\mathrm{a}_{-} \mathrm{SiO}_{x} \mathrm{NWs}$ in related devices to a certain extent. Therefore, it is imperative and crucial to explore the precise and flexible reshaping of the NW structure at room temperature. Previous studies have demonstrated that energetic electron beam (e-beam) during transmission electron microscopy (TEM) is a powerful tool which can not only in situ observe the NW structure but also athermally induce nanoscale structure instability or processing (nanoinstability or nanoprocessing). ${ }^{3-9}$ Up until now, some e-beam induced or assisted nanoprocessing in axially straight a-SiO ${ }_{x}$ NWs such as elongation, shrinkage, cutting and necking have been reported ${ }^{7-9}$ in which the length

${ }^{a}$ China-Australia Joint Laboratory for Functional Nanomaterials and Physics Department, Xiamen University, Xiamen 361005, PR China.E-mail: zhux@xmu.edu. cn

${ }^{b}$ Experiment Center of Electronic Science and Technology, School of Mathematics and Physics, Changzhou University, Changzhou 213164, PR China or diameter changes were realized. In spite of these, there is still no report found on the straightening of axially curved a-SiO NWs as purely induced by e-beam irradiation in an electron microscope. Moreover, different from the conventionallystudied axially straight NW where only positive nanocurvature is uniformly distributed, both positive and negative nanocurvatures are non-uniformly distributed over the sidewall of the axially curved NW. It is expected that such axially curved NWs would cause much more intrinsic structural instability and thus greatly different e-beam-induced kinetic behavior. However, to our best knowledge, the nanocurvature effect of a$\mathrm{SiO}_{x} \mathrm{NW}^{10,11}$ and the athermal activation effect as induced by energetic beam, ${ }^{11,12}$ which are regarded as the key factors dominating the beam-induced structure changes and atom transportations, have not been studied in such axially curved NWs. In this regard, study on the athermal reshaping of axially curved a-SiO ${ }_{x}$ NWs also makes great sense to a further fundamental understanding of new nanophenomena and concepts in low dimensional nanostructures (LDNs).

\section{Experimental}

The a-SiO ${ }_{x}$ NWs were grown by our improved chemical vapor deposition set-up where $x$ is determined to be $2.3 .{ }^{13}$ They were well-dispersed in ethanol and then deposited onto the holey carbon film of microscopy grid for TEM studies. As-prepared TEM specimens were irradiated at room temperature and the structure changes and evolution of $\mathrm{SiO}_{x} \mathrm{NWs}$ were in situ observed via a field-emission Tecnai F30 TEM operating at $300 \mathrm{kV}$ at which penetration depth of the electrons much exceeded the sample thickness or the NW diameter and energy 
deposition rate would be uniform over all the materials in the sample (see ref. 7). The irradiation was always targeted on axially curved or straight (for comparison) segment of single clean wire protruding into the open space of the holes in the carbon film of microscopy grid. Meanwhile, the two ends of the selected wires were lying on the carbon film surface and further fixed by ropes of a-SiO ${ }_{x} \mathrm{NWs}$. In each irradiation, the current density at the specimen was kept at $1 \mathrm{~A} \mathrm{~cm}^{-2}$ (electron flux: $6.25 \times$ $10^{4} \mathrm{~nm}^{-2} \mathrm{~s}^{-1}$ ) which was uniform over an area larger than the zone or NW observed. During the observation or taking a picture, the beam was spread to an around 100 times weaker intensity so that the corresponding irradiation effect can be minimized to a negligible degree and at the same time the image contrast can also be improved. Also note that during the electron irradiation, the beam was expected to heat the specimen by no more than a few degrees, ${ }^{6,7,14}$ due to its extremely large ratio of surface to volume and the dominant irradiation effect should be athermal. Therefore, it could be considered that the irradiated NW essentially remained at room temperature throughout the irradiation duration. In the experiments, the wire diameter was taken as an average value of several wire diameters which were measured at different representative positions across the wire from each micrograph. Then the length and volume of the wire segment were measured and

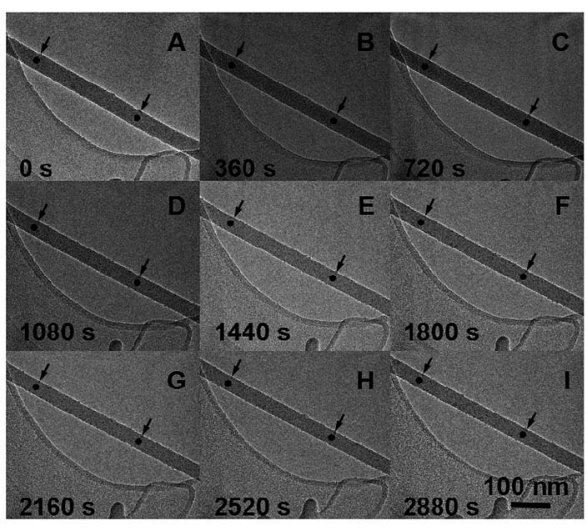

(a)

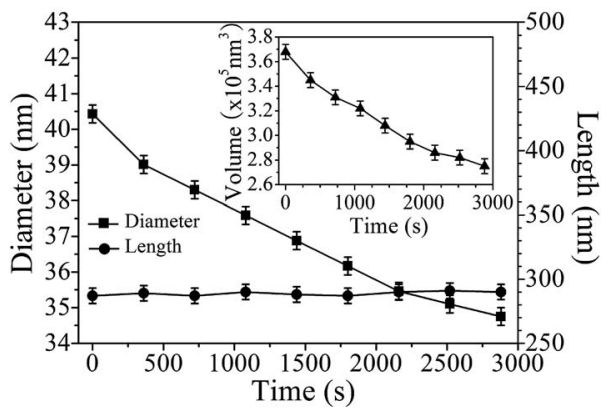

(b)

Fig. 1 (a) Sequential in situ TEM micrographs showing the typical structure changes and evolution of an axially straight $\mathrm{a}-\mathrm{SiO}_{x} \mathrm{NW}$ as induced by uniform e-beam irradiation with a current density of $1 \mathrm{~A} \mathrm{~cm}^{-2}$; (b) evolution of the diameter, length and volume of the axially straight NW segment as shown in (a) against the irradiation time.

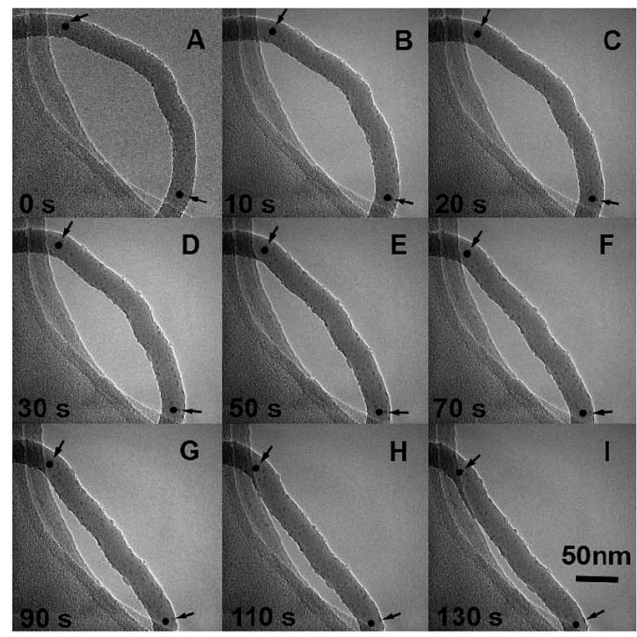

(a)

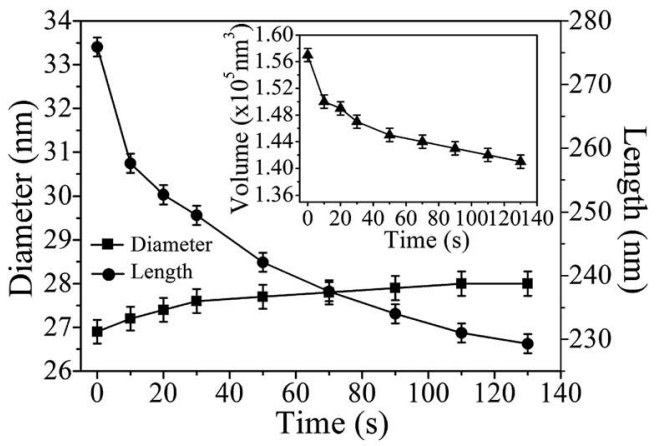

(b)

Fig. 2 (a) Sequential in situ TEM micrographs showing the typical structure changes and evolution of an axially curved $\mathrm{a}-\mathrm{SiO}_{x} \mathrm{NW}$ under the same irradiation condition as that in Fig. 1; (b) evolution of the diameter, length and volume of the axially curved NW segment as shown in (a) against the irradiation time.

calculated between two black mark dots in (a) of Fig. 1 and 2 along the curved or straight wire axis. The two black dots were carefully marked at the locations of two feature points and by further checking the relative positions of their surrounding feature points on the wire surface (see the arrows in (a) of Fig. 1 and 2).

\section{Results and discussion}

The sequential TEM micrographs in Fig. 1(a) show the typical in situ structure changes and evolution of an axially straight a-SiO $\mathrm{S}_{x}$ NW segment during uniform e-beam irradiation with a current density at $1 \mathrm{~A} \mathrm{~cm}^{-2}$. It was observed that the axially straight $\mathrm{NW}$ segment kept its perfect straight cylinder-like wire shape during the whole irradiation duration up to $2880 \mathrm{~s}$. Furthermore, since the ends were both fixed, the NW segment could not shrink in the axis direction and thus only shrunk in its diameter to minimize the surface energy with reducing its surface area. As further quantified in Fig. 1(b), with the increase of irradiation time (or electron dose), the length almost kept a constant of $288 \mathrm{~nm}$ while the diameter decreased uniformly and slowly 
from $40.4 \mathrm{~nm}$ to $34.7 \mathrm{~nm}$ with an average radial shrinking rate of $2.0 \times 10^{-3} \mathrm{~nm} \mathrm{~s}^{-1}$. Meanwhile, the volume of the NW segment decreased slowly from $3.68 \times 10^{5} \mathrm{~nm}^{3}$ to $2.75 \times 10^{5}$ $\mathrm{nm}^{3}$ with an average rate of $3.23 \times 10^{1} \mathrm{~nm}^{3} \mathrm{~s}^{-1}$ (see the inset in Fig. 1(b)). In contrast, as shown in Fig. 2(a), under the same irradiation conditions the axially curved NW segment of a circular arc-like shape turned straight intriguingly within the short duration of $130 \mathrm{~s}$ with its two ends nearly no moving or outward-protruding. Meanwhile, a notable uniform axial shrinking and radial thickening was occurring on the wire segment within the carbon film hole. As a result, the axially curved NW segment changed into an axially straight one quickly with a shorter length and a thicker diameter. As further quantitatively shown in Fig. 2(b), with the increase of irradiation time the length decreased quickly from $275.9 \mathrm{~nm}$ to $229.3 \mathrm{~nm}$ with an average axial shrinking rate of $3.6 \times 10^{-1} \mathrm{~nm} \mathrm{~s}^{-1}$ while the diameter increased much slowly from $26.9 \mathrm{~nm}$ to $28.0 \mathrm{~nm}$ with an average radial thickening rate of $8.5 \times 10^{-3} \mathrm{~nm} \mathrm{~s}^{-1}$. Meanwhile, the volume of the NW segment decreased quickly from $1.57 \times 10^{5} \mathrm{~nm}^{3}$ to $1.41 \times 10^{5} \mathrm{~nm}^{3}$ with an average rate of $1.23 \times$ $10^{2} \mathrm{~nm}^{3} \mathrm{~s}^{-1}$ (see the inset in Fig. 2(b)), which is almost 4 times that in the axially straight NW case. It was further observed that even after the above evolution in the axially straight or curved NW proceeded for a while, it would stop immediately once the irradiation was suspended. This means that the process is predominately driven by an ultrafast irradiation-induced athermal activation rather than a slow beam heating-induced thermal activation. It thus indicates a reshaping process of the axially curved NW as purely induced by the uniform e-beam irradiation. Similar irradiation on other axially curved and straight NW segments was repeated several times. We observed that the features of the structure changes and evolution were essentially the same as those shown in Fig. 1 and 2 .

As experimentally demonstrated above, the brittle a-SiO NWs exhibit remarkable reshapings such as straightening, shrinking, or thickening at room temperature under uniform e-beam irradiation. It was generally considered to be impossible to achieve such plastic deformations in glassy materials at room temperature. This is because the glass-transition temperature is as high as $1373 \mathrm{~K}$ and room temperature is too low for viscous flow to contribute significantly to the accommodation of the imposed deformation rate. ${ }^{8}$ However, with the assistance of uniform e-beam irradiation, Zheng et $a l^{8}$ have realized a superplastic elongation of axially straight silica NWs by tensile pulling; by a pure focused e-beam irradiation, we have achieved a locally-prolonged S-type deformation in an axially straight $\mathrm{a}^{-\mathrm{SiO}_{x}} \mathrm{NW} ;^{7}$ further by a pure uniform e-beam irradiation, as shown here, we have actualized a straightening, axial shrinking and radial thickening in axially curved $\mathrm{a}-\mathrm{SiO}_{x} \mathrm{NWs}$. All of these demonstrate that the energetic e-beam can athermally soften the brittle amorphous silica or $\mathrm{SiO}_{x} \mathrm{NW}$ and cause remarkable reshapings with or even without the assistance of external force.

Normally, people often resort to the traditional science concepts such as knock-on mechanism ${ }^{\mathbf{1 4}}$ and e-beam heating effect ${ }^{3,15,16}$ to explain and predict the energetic beam-induced nanophenomena. However, the knock-on mechanism and its related simulations have to be based on the equilibrium,

symmetry, periodicity and linear nature of bulk crystalline structures, or its approximations. Therefore, the knock-on mechanism and related simulations cannot differentiate the nanocurvature effect ${ }^{10,11}$ of NWs or account for the beaminduced athermal activation effect, ${ }^{\mathbf{1 1}, 12}$ both of which are of non equilibrium, amorphous-like, or nonlinear nature. This is especially true when we explain the effects associated with the fast straightening, shrinking or thickening of NW and surface atom adjustment or rearrangement at room temperature. On the other hand, due to the extremely high ratio of surface to volume of NW at the nanoscale, the e-beam irradiation is expected to heat the specimen by no more than a few degrees, ${ }^{6,7,14}$ and the dominant irradiation effect should be athermal. Moreover, as we have mentioned above, the fact that the evolution will stop immediately once the irradiation is suspended also demonstrates an ultrafast irradiation-induced athermal activation effect. In fact, our previous research ${ }^{7,9-12}$ has demonstrated that our proposed novel nanocurvature effect and energetic beam-induced athermal activation effect are universal phenomena and applicable in prediction or explanation of nanophenomena of LDNs. In the following, we attempt to reveal the above two effects on the reshaping of the $\mathrm{a}_{-} \mathrm{SiO}_{x}$ NWs especially the axially curved a-SiO ${ }_{x} \mathrm{NW}$ under uniform ebeam irradiation.

For the nanocurvature effect on an axially straight NW, we can suppose that, similar to the particle case, ${ }^{\mathbf{1 0 , 1 1}}$ when the radius of the NW approaches its atomic bond length, a positive nanocurvature on the radially-curved wire surface will become appreciable. As schematically illustrated in Fig. 3(a), however, there is no curvature in the straight wire axis direction. In contrast, for an axially curved NW as shown in Fig. 3(b), besides the radial positive nanocurvature, there are an additional positive nanocurvature and an additional negative nanocurvature further produced respectively on the convex outer sidewall and the concave inner sidewall (similar to the cavity case ${ }^{\mathbf{1 0 , 1 1}}$ ) along the curved wire axis. The positive nanocurvature

evaporation

(a)

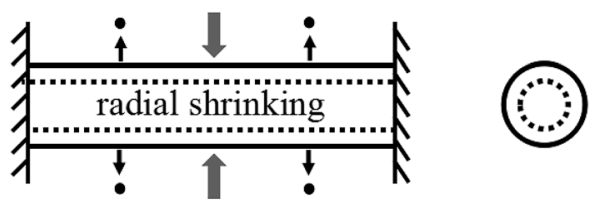

(b)

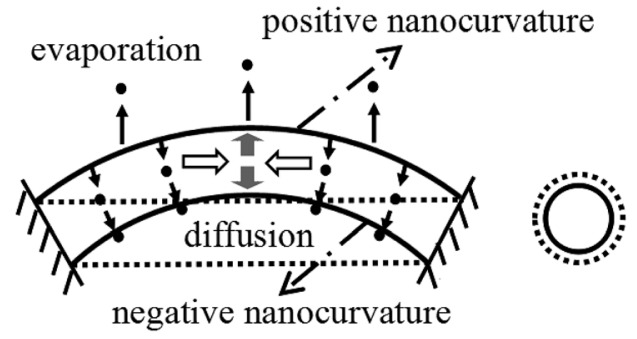

Fig. 3 Schematic diagrams showing the athermal diffusion or plastic flow and athermal evaporation of wire atoms on axially straight (a) and axially curved (b) NW segments and their resulting structure changes. 
of the radial and axial directions would cause an additional tensile stress ${ }^{\mathbf{1 0 , 1 1}}$ on the electron cloud structure of surface atoms on the convex wire sidewall. As a result, the vibration frequency of the surface atoms would be decreased and thus the "Debye temperature"10,11 would be lowered down and cause the convex wire sidewall to melt and the atoms therein to migrate or escape. Conversely, the negative nanocurvature would cause an additional compressive stress ${ }^{10,11}$ on the electron cloud structure of surface atoms on the concave wire sidewall. The compressive stress would lead to a speeding up of the vibration of the surface atoms and thus increase the "Debye temperature" and induce the concave wire sidewall to capture atoms. In this way, the positive nanocurvature and/or the negative nanocurvature would cause intrinsic structural instability tendency in the NW especially in the axially curved NW. This phenomenon was called nanocurvature effect or nanosize effect in a broad sense. ${ }^{10,11}$

Although the nanocurvatures on a NW can cause the above structural instability tendency thermodynamically, a further assistance from external excitation such as energetic beam irradiation is still needed to realize kinetically the corresponding mass transportation and structure change. This can be verified by the observation that the straightening, shrinking or thickening of the NW would stop immediately once the irradiation was suspended after the structure changes started. In the present case of energetic e-beam irradiation in TEM, we can assume that, when the beam energy deposition rate r,11,12 $^{\mathbf{9}}$ of the incident energetic beam becomes very fast, there is no enough time for the deposited energy to transfer to thermal vibration energy of atoms within a single period of the atomic vibration, and thus the mode of atom thermal vibration would be softened or the vibration of atoms would lose stability. ${ }^{11,12}$ The asinduced soft mode or instability of atomic vibration can suppress the energy barrier or even make it totally disappear. In doing so, the irradiation would cause atom transportations such as the athermal diffusion (or even athermal plastic flow) and/or athermal evaporation (or even athermal ablation) $)^{7,9}$ of the wire atoms and thus the structure changes. This phenomenon was called beam-induced athermal activation (or beaminduced atomic vibration soft mode and instability) effect, or nanotime effect in a broad sense. ${ }^{\mathbf{1 1 , 1 2}}$

For the axially straight NW as illustrated in Fig. 3(a), since the curvature is uniform over the wire surface, the wire atoms can only evaporate uniformly out of the wire surface to minimize the surface energy. With the increase of irradiation time, it thus demonstrates a uniform shrinkage in the radial direction. However, for the axially curved NW as illustrated in Fig. 3(b), the curvature distributed over the outer and inner wire surface is not uniform along the curved wire axis. In addition, there are still some minor distortions on both the outer and inner wire sidewall surface (see Fig. 2(a)). As driven by the positive and negative nanocurvature effects, the beam athermally activated or softened wire atoms on the convex locations would diffuse or even flow to the neighboring concave locations or preferentially evaporate into the vacuum (see Fig. 3(b)). Meanwhile, the preferentially-evaporated atoms from the convex locations may further deposit onto and then are absorbed by the concave locations especially the inner sidewall. With the increase of irradiation time, the concave wire sidewall is filled and the convex wire sidewall is flattened by surface atom adjustment or rearrangement. In this way, the axially curved NW segment turns straight quickly along with a decrease in its length and an increase in its diameter. As further shown in Fig. 2, the increase of wire diameter against the irradiation time seems less pronounced or to slow down at the later stage. This can be attributed to the decreasing of curvature difference between the convex and the concave sidewall along with the straightening process. In addition, in contrast to the axially straight NW, the faster decreasing of wire volume in the axially curved NW as mentioned above demonstrates a larger nanocurvature and thus with a higher resulting atom evaporation rate.

\section{Conclusions}

In this work, we studied the in situ reshapings of a-SiO ${ }_{x} \mathrm{NWs}_{\text {as }}$ purely induced by uniform e-beam irradiation at room temperature in transmission electron microscope. It was observed that the axially straight NW kept its perfect straight cylinder-like wire shape and demonstrated a uniform radial shrinkage with the increase of irradiation time. In contrast, the axially curved NW turned straight quickly accompanied with a uniform axial shrinkage and a uniform radial expansion intriguingly. The athermal diffusion and plastic flow of wire atoms in the axially curved NW indicates a direct experimental evidence for our predicted athermal activation effect as induced under uniform e-beam irradiation in condensed matter. Furthermore, the directional diffusion and flow of wire atoms from the convex wire sidewall to the nearby concave wire sidewall and the preferential evaporation of wire atoms on the convex wire sidewall demonstrate the effect of positive and negative nanocurvatures non-uniformly distributed over the axially curved NW. Such a study has important implications for the nanoinstability and nanoprocessing of future NW-based structures or devices. More importantly, the findings demonstrate that the current knock-on mechanism and e-beam heating effect are inadequate to explain these processes but our proposed nanocurvature effect and energetic beam-induced athermal activation effect obviously dominate the processes.

\section{Conflicts of interest}

There are no conflicts of interest to declare.

\section{Acknowledgements}

This work was supported by the NSFC project under grant no. 11574255, the Science and Technology Plan (Cooperation) Key Project from Fujian Province Science and Technology Department under grant no. 2014I0016, and the National Key Basic Science Research Program (973 Project) under grant no. 2007 CB936603. 


\section{References}

1 D. P. Yu, Q. L. Hang, Y. Ding, et al., Amorphous silica nanowires: Intensive blue light emitters, Appl. Phys. Lett., 1998, 73, 3076-3078.

2 A. Mondal, N. K. Singh, P. Chinnamuthu, et al., Enlarged photodetection using $\mathrm{SiO}_{x}$ nanowire arrays, IEEE Photonics Technol. Lett., 2012, 24, 2020-2023.

3 S. Y. Xu, M. L. Tian, J. G. Wang, et al., Nanometer-scale modification and welding of silicon and metallic nanowires with a high-intensity electron beam, Small, 2005, 1, 1221-1229.

4 A. V. Krasheninnikov and F. Banhart, Engineering of nanostructured carbon materials with electron or ion beams, Nat. Mater., 2007, 6, 723-733.

5 T. Aref, M. Remeika and A. Bezryadin, High-resolution nanofabrication using a highly focused electron beam, $J$. Appl. Phys., 2008, 104, 024312.

6 J. M. Zhang, L. P. You, H. Q. Ye, et al., Fabrication of ultrafine nanostructures with single-nanometer precision in a highresolution transmission electron microscope, Nanotechnology, 2007, 18, 155303.

7 X. F. Zhu, J. B. Su, Y. Wu, et al., Intriguing surface-extruded plastic flow of $\mathrm{SiO}_{\mathrm{x}}$ amorphous nanowire as athermally induced by electron beam irradiation, Nanoscale, 2014, 6, 1499-1507.
8 K. Zheng, C. Wang, Y. Q. Cheng, et al., Electron-beamassisted superplastic shaping of nanoscale amorphous silica, Nat. Commun., 2010, 1, 1-8.

9 J. B. Su and X. F. Zhu, Atom diffusion and evaporation of free-ended amorphous $\mathrm{SiO}_{\mathrm{x}}$ nanowires: nanocurvature effect and beam-induced athermal activation effect, Nanoscale Res. Lett., 2016, 11, 514.

$10 \mathrm{X}$. F. Zhu, Evidence of an antisymmetry relation between a nanocavity and a nanoparticle: a novel nanosize effect, J. Phys.: Condens. Matter, 2003, 15, L253-L261.

11 X. F. Zhu and Z. G. Wang, Nanoinstabilities as revealed by shrinkage of nanocavities in silicon during irradiation, Int. J. Nanotechnol., 2006, 3, 492-516.

$12 \mathrm{X}$. F. Zhu and Z. G. Wang, Evidence of energetic beaminduced soft mode and lattice instability: a novel nanotime effect, Chin. Phys. Lett., 2005, 22, 737-740.

13 S. L. Huang, Y. Wu, X. F. Zhu, et al., VLS growth of $\mathrm{SiO}_{\mathrm{x}}$ nanowires with a stepwise nonuniformity in diameter, $J$. Appl. Phys., 2011, 109, 084328.

14 F. Banhart, Irradiation effects in carbon nanostructures, Rep. Prog. Phys., 1999, 62, 1181-1221.

15 D. Ugarte, Curling and closure of graphitic networks under electron-beam irradiation, Nature, 1992, 359, 707709.

16 C. H. Kiang, W. A. Goddard, R. Beyers, et al., Structural modification of single-layer carbon nanotubes with an electron beam, J. Phys. Chem., 1996, 100, 3749-3752. 